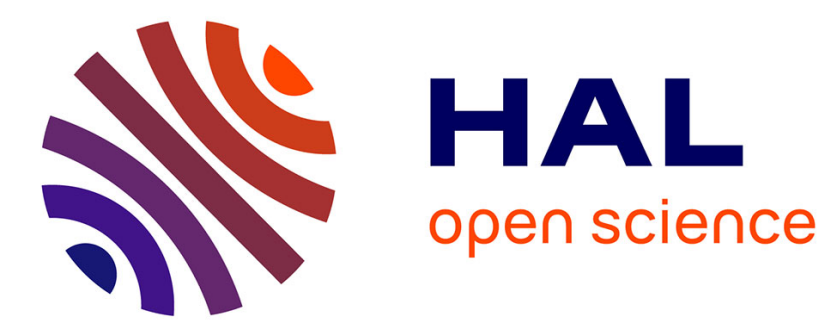

\title{
On some singular limits in damped radiation hydrodynamics
}

\author{
Xavier Blanc, B Ducomet, S Nečasová
}

\section{To cite this version:}

Xavier Blanc, B Ducomet, S Nečasová. On some singular limits in damped radiation hydrodynamics. Journal of Hyperbolic Differential Equations, 2016, 10.1142/S0219891616500089 . hal-01143250

\author{
HAL Id: hal-01143250 \\ https://hal.science/hal-01143250
}

Submitted on 17 Apr 2015

HAL is a multi-disciplinary open access archive for the deposit and dissemination of scientific research documents, whether they are published or not. The documents may come from teaching and research institutions in France or abroad, or from public or private research centers.
L'archive ouverte pluridisciplinaire HAL, est destinée au dépôt et à la diffusion de documents scientifiques de niveau recherche, publiés ou non, émanant des établissements d'enseignement et de recherche français ou étrangers, des laboratoires publics ou privés. 


\title{
On some singular limits in damped radiation hydrodynamics
}

\author{
X. Blanc, B. Ducomet, S. Nečasová
}

\begin{abstract}
We consider the Cauchy problem for the 3D Euler system with damping coupled to radiation through two singular limits. Assuming suitable smallness hypotheses for the data, we prove that each of these two problems admits a unique smooth solution.
\end{abstract}

Keywords: compressible, Euler, damping, radiation.

AMS subject classification: 35Q30, 76N10

\section{Introduction}

After the study of Buet and Després [5] we consider two singular limits for a compressible inviscid radiative flow where the motion of the fluid is given by the Euler system with damping for the evolution of the density $\varrho=\varrho(t, x)$, the velocity field $\vec{u}=\vec{u}(t, x)$, and the absolute temperature $\vartheta=\vartheta(t, x)$ as functions of the time $t$ and the Eulerian spatial coordinate $x \in \mathbb{R}^{3}$.

In the first regime (equilibrium diffusion), the effect of radiation is incorporated in the state functions $p$ (pressure) and $e$ (internal energy). In the second regime (non equilibrium diffusion), the radiation appears through an extra equation of parabolic type for the radiative temperature which is a priori different from the matter temperature.

More specifically, in the equilibrium case, the system of equations to be studied for the three unknowns $(\varrho, \vec{u}, \vartheta)$ reads

$$
\begin{gathered}
\partial_{t} \varrho+\operatorname{div}_{x}(\varrho \vec{u})=0, \\
\partial_{t}(\varrho \vec{u})+\operatorname{div}_{x}(\varrho \vec{u} \otimes \vec{u})+\nabla_{x}\left(p+p_{r}\right)+\nu \vec{u}=0, \\
\partial_{t}\left(\varrho E+E_{r}\right)+\operatorname{div}_{x}\left[\left(\varrho E+E_{r}\right) \vec{u}+\left(p+p_{r}\right) \vec{u}\right]=\operatorname{div}_{x}\left(\kappa \nabla_{x} \vartheta\right)+\operatorname{div}_{x}\left(\frac{1}{3 \sigma_{a}} \nabla_{x} E_{r}\right),
\end{gathered}
$$

where $E=\frac{1}{2}|\vec{u}|^{2}+e(\varrho, \vartheta), E_{r}=a \vartheta^{4}$ and $p_{r}=\frac{a}{3} \vartheta^{4}$.

In the non-equilibrium case, the system of equations for the four unknowns $\left(\varrho, \vec{u}, \vartheta, E_{r}\right)$ is

$$
\begin{gathered}
\partial_{t} \varrho+\operatorname{div}_{x}(\varrho \vec{u})=0 \\
\partial_{t}(\varrho \vec{u})+\operatorname{div}_{x}(\varrho \vec{u} \otimes \vec{u})+\nabla_{x}\left(p+p_{r}\right)+\nu \vec{u}=0, \\
\partial_{t}(\varrho E)+\operatorname{div}_{x}((\varrho E+p) \vec{u})+\vec{u} \cdot \nabla_{x} p_{r}=\operatorname{div}_{x}\left(\kappa \nabla_{x} \vartheta\right)-\sigma_{a}\left(a \vartheta^{4}-E_{r}\right), \\
\partial_{t} E_{r}+\operatorname{div}_{x}\left(E_{r} \vec{u}\right)+p_{r} \operatorname{div}_{x} \vec{u}=\operatorname{div}_{x}\left(\frac{1}{3 \sigma_{s}} \nabla_{x} E_{r}\right)-\sigma_{a}\left(E_{r}-a \vartheta^{4}\right),
\end{gathered}
$$


where $E=\frac{1}{2}|\vec{u}|^{2}+e(\varrho, \vartheta), E_{r}$ is the radiative energy related to the temperature of radiation $T_{r}$ by $E_{r}=a T_{r}^{4}$ and $p_{r}$ is the radiative pressure given by $p_{r}=\frac{1}{3} a T_{r}^{4}=\frac{1}{3} E_{r}$, with $a>0$.

Systems (1.1) - (1.3) and (1.4) - (1.7) can be viewed as singular limits in radiation hydrodynamics in two limit diffusion regimes. Such systems (when damping is absent) have been investigated by Lowrie, Morel and Hittinger [25] and more recently by Buet and Després [5].

In a recent paper, Lin and Goudon [24] consider an equilibrium diffusion system close to (1.1 1.3). Using a similar analysis and additional arguments introduced by Beauchard and Zuazua [3], our goal is to prove global existence of solutions for the system (1.1) - (1.3) (resp. (1.4) - (1.7)) when data are sufficiently close to an equilibrium state $(\bar{\varrho}, 0, \bar{\theta})$ (resp. $\left(\bar{\varrho}, 0, \bar{\vartheta}, \overline{E_{r}}\right)$ ), with $\bar{\varrho}>0$, $\bar{\vartheta}>0$ and $\overline{E_{r}}>0$.

\section{Hypotheses}

Hypotheses imposed on constitutive relations and transport coefficients are motivated by the general (local) existence theory for the Euler-Fourier system developed in [30,31] (see also [12, Chapter 3] for the Navier-Stokes-Fourier framework) and reasonable physical assumptions for the radiative part $[27,29]$.

In our simplified setting, transport coefficients $\kappa, \sigma_{a}, \sigma_{s}$ and the Planck's coefficient $a$ are supposed to be fixed positive numbers.

The damping with coefficient $\nu>0$ of Darcy type can be interpreted here as a diffusion of a light gas into a heavy one.

We consider the pressure in the form

$$
p(\varrho, \vartheta)=\vartheta^{5 / 2} P\left(\frac{\varrho}{\vartheta^{3 / 2}}\right), a>0,
$$

where $P:[0, \infty) \rightarrow[0, \infty)$ is a given function with the following properties:

$$
\begin{gathered}
P \in C^{1}[0, \infty), P(0)=0, P^{\prime}(Z)>0 \text { for all } Z \geq 0, \\
0<\frac{\frac{5}{3} P(Z)-P^{\prime}(Z) Z}{Z}<c \text { for all } Z \geq 0, \\
\lim _{Z \rightarrow \infty} \frac{P(Z)}{Z^{5 / 3}}=p_{\infty}>0 .
\end{gathered}
$$

After Maxwell's relations, the specific internal energy $e$ is

$$
e(\varrho, \vartheta)=\frac{3}{2} \vartheta\left(\frac{\vartheta^{3 / 2}}{\varrho}\right) P\left(\frac{\varrho}{\vartheta^{3 / 2}}\right),
$$

and the associated specific entropy reads

$$
s(\varrho, \vartheta)=M\left(\frac{\varrho}{\vartheta^{3 / 2}}\right),
$$

with

$$
M^{\prime}(Z)=-\frac{3}{2} \frac{\frac{5}{3} P(Z)-P^{\prime}(Z) Z}{Z^{2}}<0 .
$$

\section{Main results}

We are going to prove that, under the above structural assumptions on the equation of state, system (1.1)-(1.2)-(1.3) on the one hand, and system (1.4)-(1.5)-(1.6) on the other hand, have global smooth solutions close to any equilibrium state. 
Theorem 3.1. Let $(\bar{\varrho}, 0, \bar{\vartheta})$ be a constant state with $\bar{\varrho}>0, \bar{\vartheta}>0$. Consider $d>7 / 2$. There exists $\varepsilon>0$ such that, for any initial state $\left(\varrho_{0}, \vec{u}_{0}, \vartheta_{0}\right)$ satisfying

$$
\left\|\left(\varrho_{0}, \vec{u}_{0}, \vartheta_{0}\right)-(\bar{\varrho}, 0, \bar{\vartheta})\right\|_{H^{d}\left(\mathbb{R}^{3}\right)} \leq \varepsilon,
$$

there exists a unique global solution $(\varrho, \vec{u}, \vartheta)$ to (1.1)-(1.2)-(1.3), such that

$(\varrho-\bar{\varrho}, \vec{u}, \vartheta-\bar{\vartheta}) \in C\left([0,+\infty) ; H^{d}\left(\mathbb{R}^{3}\right)\right)$. In addition, this solution satisfies the following energy inequality:

$$
\begin{array}{r}
\|(\varrho(t)-\bar{\varrho}, \vec{u}(t), \vartheta(t)-\bar{\vartheta})\|_{H^{d}\left(\mathbb{R}^{3}\right)}+\int_{0}^{t}\left(\left\|\nabla_{x}(\varrho, \vec{u}, \vartheta)(s)\right\|_{H^{d-1}\left(\mathbb{R}^{3}\right)}^{2}+\left\|\nabla_{x} \vartheta(s)\right\|_{H^{d}}^{2}\left(\mathbb{R}^{3}\right)\right) d s \\
\leq C\left\|\left(\varrho_{0}-\bar{\varrho}, 0, \vartheta_{0}-\bar{\vartheta}\right)\right\|_{H^{d}\left(\mathbb{R}^{3}\right)}^{2}, \quad(3.2)
\end{array}
$$

for some constant $C>0$ which does not depend on $t$.

The same result holds in the case of system (1.4)-(1.5)-(1.6)-(1.7):

Theorem 3.2. Let $\left(\bar{\varrho}, 0, \bar{\vartheta}, \overline{E_{r}}\right)$ be a constant state with $\bar{\varrho}>0, \bar{\vartheta}>0, \overline{E_{r}}>0$. Consider $d>7 / 2$. There exists $\varepsilon>0$ such that, for any initial state $\left(\varrho_{0}, \vec{u}_{0}, \vartheta_{0}, E_{r}^{0}\right)$ satisfying

$$
\left\|\left(\varrho_{0}, \vec{u}_{0}, \vartheta_{0}, E_{r}^{0}\right)-\left(\bar{\varrho}, 0, \bar{\vartheta}, \overline{E_{r}}\right)\right\|_{H^{d}\left(\mathbb{R}^{3}\right)} \leq \varepsilon
$$

there exists a unique global solution $\left(\varrho, \vec{u}, \vartheta, E_{r}\right)$ to $(1.4)-(1.5)-(1.6)-(1.7)$, such that $\left(\varrho-\bar{\varrho}, \vec{u}, \vartheta-\bar{\vartheta}, \overline{E_{r}}-E_{r}\right) \in$ $C\left([0,+\infty) ; H^{d}\left(\mathbb{R}^{3}\right)\right)$. In addition, this solution satisfies the following energy inequality:

$$
\begin{aligned}
&\left\|\left(\varrho(t)-\bar{\varrho}, \vec{u}(t), \vartheta(t)-\bar{\vartheta}, E_{r}(t)-\overline{E_{r}}\right)\right\|_{H^{d}\left(\mathbb{R}^{3}\right)}+\int_{0}^{t}\left\|\nabla_{x}\left(\varrho, \vec{u}, \vartheta, E_{r}\right)(s)\right\|_{H^{d-1}\left(\mathbb{R}^{3}\right)}^{2} d s \\
&+ \int_{0}^{t}\left(\left\|\nabla_{x} \vartheta(s)\right\|_{H^{d}\left(\mathbb{R}^{3}\right)}^{2}+\left\|\nabla_{x} E_{r}(s)\right\|_{H^{d}\left(\mathbb{R}^{3}\right)}^{2}\right) d s \\
& \leq C\left\|\left(\varrho_{0}-\bar{\varrho}, 0, \vartheta_{0}-\bar{\vartheta}, E_{r}^{0}-\overline{E_{r}}\right)\right\|_{H^{d}}^{2}\left(\mathbb{R}^{3}\right),
\end{aligned}
$$

for some constant $C>0$ which does not depend on $t$.

As we will see in Section 4 below, the structure of these two systems is very similar. Indeed, imposing the equality $E_{r}=a \vartheta^{4}$ in the system (1.4)-(1.5)-(1.6)-(1.7), one finds (1.1)-(1.2)-(1.3). Therefore, the proofs of the above results are very similar. Hence, we will only give the proof of Theorem 3.2, which is a generalization of that of Theorem 3.1.

\section{The linearized systems}

\subsection{The equilibrium limit}

Equation (1.3) rewrites

$$
\varrho C_{v}\left(\partial_{t} \vartheta+\vec{u} \cdot \nabla_{x} \vartheta\right)+\vartheta p_{\vartheta} \operatorname{div}_{x} \vec{u}+\nu|\vec{u}|^{2}=-p_{r} \operatorname{div}_{x} \vec{u}+\operatorname{div}_{x}\left(\kappa \nabla_{x} \vartheta\right)+\operatorname{div}_{x}\left(\frac{1}{3 \sigma_{a}} \nabla_{x} E_{r}\right) .
$$

Linearizing the system $(1.1)-(1.3)$ around the constant state $(\bar{\varrho}, 0, \bar{\vartheta})$ and putting $\varrho=r+\bar{\varrho}$, $\vartheta=T+\bar{\vartheta}$ and $E_{r}=e_{r}+\bar{E}_{r}$, we get

$$
\partial_{t} r+\bar{\varrho} \operatorname{div}_{x} \vec{u}=0
$$




$$
\begin{gathered}
\partial_{t} \vec{u}+\frac{\bar{p}_{\varrho}}{\bar{\varrho}} \nabla_{x} r+\frac{1}{\bar{\varrho}}\left(\bar{p}_{\vartheta}+\frac{4 a \bar{\vartheta}^{3}}{3}\right) \nabla_{x} T+\nu \vec{u}=0, \\
\partial_{t} T+\frac{\bar{\vartheta} \bar{p}_{\vartheta}}{\bar{\varrho}_{\bar{C}}} \operatorname{div}_{x} \vec{u}=\operatorname{div}_{x}\left(\frac{1}{\bar{\varrho} \bar{C}_{v}}\left(\kappa+\frac{4 a \bar{\vartheta}^{3}}{3 \sigma_{a}}\right) \nabla_{x} T\right),
\end{gathered}
$$

where $\bar{C}_{v}=e_{\vartheta}(\bar{\vartheta})$.

$$
\begin{gathered}
\text { Using the vector notation } U:=\left(\begin{array}{c}
r \\
u_{1} \\
u_{2} \\
u_{3} \\
T
\end{array}\right) \text {, the linearized system (1.1) - (1.3) rewrites } \\
\partial_{t} U+\sum_{j=1}^{3} A_{j} \partial_{j} U=D \Delta U-B U
\end{gathered}
$$

with

$$
A_{1}:=\left(\begin{array}{ccccc}
0 & \bar{\varrho} & 0 & 0 & 0 \\
\alpha & 0 & 0 & 0 & \beta \\
0 & 0 & 0 & 0 & 0 \\
0 & 0 & 0 & 0 & 0 \\
0 & \gamma & 0 & 0 & 0
\end{array}\right), A_{2}:=\left(\begin{array}{ccccc}
0 & 0 & \bar{\varrho} & 0 & 0 \\
0 & 0 & 0 & 0 & 0 \\
\alpha & 0 & 0 & 0 & \beta \\
0 & 0 & 0 & 0 & 0 \\
0 & 0 & \gamma & 0 & 0
\end{array}\right), A_{3}:=\left(\begin{array}{ccccc}
0 & 0 & 0 & \bar{\varrho} & 0 \\
0 & 0 & 0 & 0 & 0 \\
0 & 0 & 0 & 0 & 0 \\
\alpha & 0 & 0 & 0 & \beta \\
0 & 0 & 0 & \gamma & 0
\end{array}\right),
$$

and

where

$$
B:=\left(\begin{array}{ccccc}
0 & 0 & 0 & 0 & 0 \\
0 & \nu & 0 & 0 & 0 \\
0 & 0 & \nu & 0 & 0 \\
0 & 0 & 0 & \nu & 0 \\
0 & 0 & 0 & 0 & 0
\end{array}\right), D:=\left(\begin{array}{ccccc}
0 & 0 & 0 & 0 & 0 \\
0 & 0 & 0 & 0 & 0 \\
0 & 0 & 0 & 0 & 0 \\
0 & 0 & 0 & 0 & 0 \\
0 & 0 & 0 & 0 & \delta
\end{array}\right)
$$

$$
\alpha=\frac{\bar{p}_{\varrho}}{\bar{\varrho}}, \quad \beta=\frac{1}{\bar{\varrho}}\left(\bar{p}_{\vartheta}+\frac{4 a \bar{\vartheta}^{3}}{3}\right), \quad \gamma=\frac{\bar{\vartheta} \bar{p}_{\vartheta}}{\bar{\varrho} \bar{C}_{v}}, \quad \delta=\frac{1}{\bar{\varrho} \bar{C}_{v}}\left(\kappa+\frac{4 a \bar{\vartheta}^{3}}{3 \sigma_{a}}\right) .
$$

In order to apply the Kreiss theory we have to put the system (4.5) in a symmetric form [4]. For that purpose it is sufficient to consider a diagonal symmetrizer

$$
\widetilde{A}_{0}=\left(\begin{array}{ccccc}
a & 0 & 0 & 0 & 0 \\
0 & b & 0 & 0 & 0 \\
0 & 0 & b & 0 & 0 \\
0 & 0 & 0 & b & 0 \\
0 & 0 & 0 & 0 & c
\end{array}\right)
$$

with the relations $a=\frac{\alpha \gamma}{\bar{\rho} \beta}$ and $b=\frac{\gamma}{\beta}$ and choosing $c=1$ in order to keep the parabolic term unchanged.

Multiplying the first equation (4.5) by $\widetilde{A}_{0}$ on the left, we get

$$
\widetilde{A}_{0} \partial_{t} U+\sum_{j=1}^{3} \widetilde{A}_{j} \partial_{j} U=\widetilde{D} \Delta U-\widetilde{B} U,
$$

where the matrices $\widetilde{A}_{j}=\widetilde{A}_{0} A_{j}$ and $\widetilde{B}=\widetilde{A}_{0} B$ are symmetric for $j=0,1,2,3$, that is,

$$
\widetilde{A}_{1}:=\left(\begin{array}{ccccc}
0 & \frac{\alpha \gamma}{\beta} & 0 & 0 & 0 \\
\frac{\alpha \gamma}{\beta} & 0 & 0 & 0 & \gamma \\
0 & 0 & 0 & 0 & 0 \\
0 & 0 & 0 & 0 & 0 \\
0 & \gamma & 0 & 0 & 0
\end{array}\right), \widetilde{A}_{2}:=\left(\begin{array}{ccccc}
0 & 0 & \frac{\alpha \gamma}{\beta} & 0 & 0 \\
0 & 0 & 0 & 0 & 0 \\
\frac{\alpha \gamma}{\beta} & 0 & 0 & 0 & \gamma \\
0 & 0 & 0 & 0 & 0 \\
0 & 0 & \gamma & 0 & 0
\end{array}\right), \widetilde{A}_{3}:=\left(\begin{array}{ccccc}
0 & 0 & 0 & \frac{\alpha \gamma}{\beta} & 0 \\
0 & 0 & 0 & 0 & 0 \\
0 & 0 & 0 & 0 & 0 \\
\frac{\alpha \gamma}{\beta} & 0 & 0 & 0 & \gamma \\
0 & 0 & 0 & \gamma & 0
\end{array}\right),
$$


and

$$
\widetilde{B}:=\left(\begin{array}{ccccc}
0 & 0 & 0 & 0 & 0 \\
0 & \frac{\nu \gamma}{\beta} & 0 & 0 & 0 \\
0 & 0 & \frac{\nu \gamma}{\beta} & 0 & 0 \\
0 & 0 & 0 & \frac{\nu \gamma}{\beta} & 0 \\
0 & 0 & 0 & 0 & 0
\end{array}\right), \widetilde{D}:=\left(\begin{array}{ccccc}
0 & 0 & 0 & 0 & 0 \\
0 & 0 & 0 & 0 & 0 \\
0 & 0 & 0 & 0 & 0 \\
0 & 0 & 0 & 0 & 0 \\
0 & 0 & 0 & 0 & \delta
\end{array}\right),
$$

where $\alpha, \beta \gamma$ and $\delta$ are defined by (4.6).

The hyperbolic-parabolic system (4.7) is now symmetric.

\subsection{The non-equilibrium limit}

Equation (1.6) rewrites

$$
\varrho C_{v}\left(\partial_{t} \vartheta+\vec{u} \cdot \nabla_{x} \vartheta\right)+\vartheta p_{\vartheta} \operatorname{div}_{x} \vec{u}=-p_{r} \operatorname{div}_{x} \vec{u}+\operatorname{div}_{x}\left(\kappa \nabla_{x} \vartheta\right)-\sigma_{a}\left(a \vartheta^{4}-E_{r}\right)-\nu|\vec{u}|^{2} .
$$

Linearizing the system (1.4) - (1.6) around the constant state $\left(\bar{\varrho}, 0, \bar{\vartheta}, \bar{E}_{r}\right)$ with the compatibility condition $\bar{E}_{r}=a \bar{\vartheta}^{4}$ and putting $\varrho=r+\bar{\varrho}, \vartheta=T+\bar{\vartheta}$ and $E_{r}=e_{r}+\bar{E}_{r}$ we get

$$
\begin{gathered}
\partial_{t} r+\bar{\varrho} \operatorname{div}_{x} \vec{u}=0, \\
\partial_{t} \vec{u}+\frac{\bar{p}_{\varrho}}{\bar{\varrho}} \nabla_{x} r+\frac{\bar{p}_{\vartheta}}{\bar{\varrho}} \nabla_{x} T+\frac{1}{3 \bar{\varrho}} \nabla_{x} e_{r}+\nu \vec{u}=0, \\
\partial_{t} T+\frac{\bar{\vartheta}_{\bar{p}_{\vartheta}} \overline{\bar{C}}_{v}}{\operatorname{div}_{x} \vec{u}}=\operatorname{div}_{x}\left(\frac{\kappa}{\bar{\varrho} \bar{C}_{v}} \nabla_{x} T\right)-\frac{\sigma_{a}}{\bar{\varrho} \bar{C}_{v}}\left(4 a \bar{\vartheta}^{3} T-e_{r}\right), \\
\partial_{t} e_{r}+\frac{4}{3} \bar{E}_{r} \operatorname{div}_{x} \vec{u}=\operatorname{div}_{x}\left(\frac{1}{3 \sigma_{s}} \nabla_{x} e_{r}\right)-\sigma_{a}\left(e_{r}-4 a \bar{\vartheta}^{3} T\right) . \\
\text { Using the vector notation } U:=\left(\begin{array}{c}
r \\
u_{1} \\
u_{2} \\
u_{3} \\
T \\
e_{r}
\end{array}\right), \text { the linearized system (4.9) - (4.12) rewrites } \\
\partial_{t} U+\sum_{j=1}^{3} A_{j} \partial_{j} U=D \Delta U-B U,
\end{gathered}
$$

with

$$
\begin{gathered}
A_{1}:=\left(\begin{array}{cccccc}
0 & \bar{\varrho} & 0 & 0 & 0 & 0 \\
\alpha & 0 & 0 & 0 & \beta & \frac{1}{3 \bar{\varrho}} \\
0 & 0 & 0 & 0 & 0 & 0 \\
0 & 0 & 0 & 0 & 0 & 0 \\
0 & \gamma & 0 & 0 & 0 & 0 \\
0 & \delta & 0 & 0 & 0 & 0
\end{array}\right), \quad A_{2}:=\left(\begin{array}{ccccccccc}
0 & 0 & \varrho & 0 & 0 & 0 \\
0 & 0 & 0 & 0 & 0 & 0 \\
\alpha & 0 & 0 & 0 & \beta & \frac{1}{3 \bar{\varrho}} \\
0 & 0 & 0 & 0 & 0 & 0 \\
0 & 0 & \gamma & 0 & 0 & 0 \\
0 & 0 & \delta & 0 & 0 & 0
\end{array}\right), \\
A_{3}:=\left(\begin{array}{cccccc}
0 & 0 & 0 & \bar{\varrho} & 0 & 0 \\
0 & 0 & 0 & 0 & 0 & 0 \\
0 & 0 & 0 & 0 & 0 & 0 \\
\alpha & 0 & 0 & 0 & \beta & \frac{1}{3 \bar{\varrho}} \\
0 & 0 & 0 & \gamma & 0 & 0 \\
0 & 0 & 0 & \delta & 0 & 0
\end{array}\right),
\end{gathered}
$$


and

$$
D:=\left(\begin{array}{cccccc}
0 & 0 & 0 & 0 & 0 & 0 \\
0 & 0 & 0 & 0 & 0 & 0 \\
0 & 0 & 0 & 0 & 0 & 0 \\
0 & 0 & 0 & 0 & 0 & 0 \\
0 & 0 & 0 & 0 & \mu & 0 \\
0 & 0 & 0 & 0 & 0 & \nu
\end{array}\right), B:=\left(\begin{array}{cccccc}
0 & 0 & 0 & 0 & 0 & 0 \\
0 & \nu & 0 & 0 & 0 & 0 \\
0 & 0 & \nu & 0 & 0 & 0 \\
0 & 0 & 0 & \nu & 0 & 0 \\
0 & 0 & 0 & 0 & \zeta & -\eta \\
0 & 0 & 0 & 0 & -\pi & \sigma_{a}
\end{array}\right)
$$

where

$$
\begin{gathered}
\alpha=\frac{\bar{p}_{\varrho}}{\bar{\varrho}}, \quad \beta=\frac{\bar{p}_{\vartheta}}{\bar{\varrho}}, \quad \gamma=\frac{\bar{\vartheta} \bar{p}_{\vartheta}}{\bar{\varrho} \bar{C}_{v}}, \quad \delta=\frac{4}{3} \bar{E}_{r}, \quad \mu=\frac{\kappa}{\bar{\varrho} \bar{C}_{v}}, \\
\tau=\frac{1}{3 \sigma_{s}}, \quad \zeta=\frac{4 a \sigma_{a} \bar{\vartheta}^{3}}{\bar{\varrho} \bar{C}_{v}}, \quad \eta=\frac{\sigma_{a}}{\bar{\varrho}_{\bar{C}}}, \quad \pi=4 a \sigma_{a} \bar{\vartheta}^{3} .
\end{gathered}
$$

In order to apply the Kreiss theory we have to put the system (4.13) in a symmetric form [4]. For that purpose it is sufficient to consider a diagonal symmetrizer

$$
\widetilde{A}_{0}=\left(\begin{array}{cccccc}
a & 0 & 0 & 0 & 0 & 0 \\
0 & b & 0 & 0 & 0 & 0 \\
0 & 0 & b & 0 & 0 & 0 \\
0 & 0 & 0 & b & 0 & 0 \\
0 & 0 & 0 & 0 & c & 0 \\
0 & 0 & 0 & 0 & 0 & d
\end{array}\right)
$$

We get the relations $a=\frac{\alpha}{\bar{\varrho}} b, c=\frac{\beta}{\gamma} b$ and $d=\frac{1}{3 \bar{\varrho} \delta} b$.

Multiplying the first equation (4.13) by $\widetilde{A}_{0}$ on the left, we get

$$
\widetilde{A}_{0} \partial_{t} U+\sum_{j=1}^{3} \widetilde{A}_{j} \partial_{j} U=\widetilde{D} \Delta U-\widetilde{B} U,
$$

where the matrices $\widetilde{A}_{j}=\widetilde{A}_{0} A_{j}$ are symmetric, for all $j=1,2,3$. More specifically,

$$
\begin{gathered}
\widetilde{A}_{1}:=\left(\begin{array}{cccccc}
0 & \alpha b & 0 & 0 & 0 & 0 \\
\alpha b & 0 & 0 & 0 & \beta b & \frac{b}{3 \bar{\varrho}} \\
0 & 0 & 0 & 0 & 0 & 0 \\
0 & 0 & 0 & 0 & 0 & 0 \\
0 & \beta b & 0 & 0 & 0 & 0 \\
0 & \frac{b}{3 \bar{\varrho}} & 0 & 0 & 0 & 0
\end{array}\right), \quad \widetilde{A}_{2}:=\left(\begin{array}{cccccccc}
0 & 0 & \alpha b & 0 & 0 & 0 \\
0 & 0 & 0 & 0 & 0 & 0 \\
\alpha b & 0 & 0 & 0 & \beta b & \frac{b}{3 \bar{\varrho}} \\
0 & 0 & 0 & 0 & 0 & 0 \\
0 & 0 & \beta b & 0 & 0 & 0 \\
0 & 0 & \frac{b}{3 \bar{\varrho}} & 0 & 0 & 0
\end{array}\right), \\
\widetilde{A}_{3}:=\left(\begin{array}{cccccc}
0 & 0 & 0 & \alpha b & 0 & 0 \\
0 & 0 & 0 & 0 & 0 & 0 \\
0 & 0 & 0 & 0 & 0 & 0 \\
\alpha b & 0 & 0 & 0 & \beta b & \frac{b}{3 \bar{\varrho}} \\
0 & 0 & 0 & \beta b & 0 & 0 \\
0 & 0 & 0 & \frac{b}{3 \bar{\varrho}} & 0 & 0
\end{array}\right) .
\end{gathered}
$$

The hyperbolic part system (4.14) is now symmetric while its symmetric dissipative part is given by

$$
\widetilde{D}:=\left(\begin{array}{cccccc}
0 & 0 & 0 & 0 & 0 & 0 \\
0 & 0 & 0 & 0 & 0 & 0 \\
0 & 0 & 0 & 0 & 0 & 0 \\
0 & 0 & 0 & 0 & 0 & 0 \\
0 & 0 & 0 & 0 & c \mu & 0 \\
0 & 0 & 0 & 0 & 0 & d \tau
\end{array}\right), \widetilde{B}:=\left(\begin{array}{cccccc}
0 & 0 & 0 & 0 & 0 & 0 \\
0 & b \nu & 0 & 0 & 0 & 0 \\
0 & 0 & b \nu & 0 & 0 & 0 \\
0 & 0 & 0 & b \nu & 0 & 0 \\
0 & 0 & 0 & 0 & c \zeta & -c \eta \\
0 & 0 & 0 & 0 & -d \pi & d \sigma_{a}
\end{array}\right)
$$


where one checks the relation $c \eta=d \pi=\frac{\sigma_{a}}{\bar{\varrho} \bar{\vartheta}} b$ and the positiveness condition of $\widetilde{B}$

$$
{ }^{t} X \widetilde{B} X>0, \text { for any vector } X \in \mathbb{R}^{6} \text {. }
$$

Applying the Fourier transform in $x$ to (4.14) we get

$$
\widetilde{A}_{0} \partial_{t} \widehat{U}+i \sum_{j=1}^{3} \xi_{j} \widetilde{A}_{j} \widehat{U}=-|\xi|^{2} \widetilde{D} \widehat{U}-\widetilde{B} \widehat{U}
$$

or

$$
\widetilde{A}_{0} \partial_{t} \widehat{U}=E(\xi) \widehat{U}
$$

with

$$
E(\xi)=-B(\xi)-i A(\xi)
$$

where

$$
A(\xi)=\sum_{j=1}^{3} \xi_{j} \widetilde{A}_{j}=\left(\begin{array}{cccccc}
0 & a \bar{\varrho} \xi_{1} & a \bar{\varrho} \xi_{2} & a \bar{\varrho} \xi_{3} & 0 & 0 \\
b \alpha \xi_{1} & 0 & 0 & 0 & b \beta \xi_{1} & \frac{b}{3 \bar{\varrho}} \xi_{1} \\
b \alpha \xi_{2} & 0 & 0 & 0 & b \beta \xi_{2} & \frac{b}{3 \bar{b}} \xi_{2} \\
b \alpha \xi_{3} & 0 & 0 & 0 & b \beta \xi_{3} & \frac{b}{3 \bar{\varrho}} \xi_{3} \\
0 & c \gamma \xi_{1} & c \gamma \xi_{2} & c \gamma \xi_{3} & 0 & 0 \\
0 & d \delta \xi_{1} & d \delta \xi_{2} & d \delta \xi_{3} & 0 & 0
\end{array}\right)
$$

and

$$
B(\xi):=\widetilde{B}+|\xi|^{2} \widetilde{D}=\left(\begin{array}{cccccc}
0 & 0 & 0 & 0 & 0 & 0 \\
0 & b \nu & 0 & 0 & 0 & 0 \\
0 & 0 & b \nu & 0 & 0 & 0 \\
0 & 0 & 0 & b \nu & 0 & 0 \\
0 & 0 & 0 & 0 & c\left(\mu|\xi|^{2}+\zeta\right) & -c \eta \\
0 & 0 & 0 & 0 & -d \pi & d\left(\tau|\xi|^{2}+\sigma_{a}\right)
\end{array}\right) .
$$

Choosing $b=3 \bar{\varrho}$, we get

$$
a=\frac{3 \bar{p}_{\vartheta}}{\varrho}, \quad c=\frac{3 \bar{C}_{v} \bar{\varrho}}{\bar{\vartheta}}, \quad d=\frac{3}{4 \bar{E}_{r}},
$$

so

$$
A(\xi)=\left(\begin{array}{cccccc}
0 & 3 \bar{p}_{\varrho} \xi_{1} & 3 \bar{p}_{\varrho} \xi_{2} & 3 \bar{p}_{\varrho} \xi_{3} & 0 & 0 \\
3 \bar{p}_{\varrho} \xi_{1} & 0 & 0 & 0 & 3 \bar{p}_{\vartheta} \xi_{1} & \xi_{1} \\
3 \bar{p}_{\varrho} \xi_{2} & 0 & 0 & 0 & 3 \bar{p}_{\vartheta} \xi_{2} & \xi_{2} \\
3 \bar{p}_{\varrho} \xi_{3} & 0 & 0 & 0 & 3 \bar{p}_{\vartheta} \xi_{3} & \xi_{3} \\
0 & 3 \bar{p}_{\vartheta} \xi_{1} & 3 \bar{p}_{\vartheta} \xi_{2} & 3 \bar{p}_{\vartheta} \xi_{3} & 0 & 0 \\
0 & \xi_{1} & \xi_{2} & \xi_{3} & 0 & 0
\end{array}\right)
$$

and

$$
B(\xi):=\left(\begin{array}{cccccc}
0 & 0 & 0 & 0 & 0 & 0 \\
0 & 3 \bar{\varrho} \nu & 0 & 0 & 0 & 0 \\
0 & 0 & 3 \bar{\varrho} \nu & 0 & 0 & 0 \\
0 & 0 & 0 & 3 \bar{\varrho} \nu & 0 & 0 \\
0 & 0 & 0 & 0 & \frac{3 \bar{\kappa}}{\bar{\vartheta}}|\xi|^{2}+12 a \sigma_{a} \bar{\vartheta}^{2} & -\frac{3 \sigma_{a}}{\bar{\vartheta}} \\
0 & 0 & 0 & 0 & -\frac{3 \sigma_{a}}{\bar{\vartheta}} & \frac{1}{4 a \sigma_{s} \bar{\vartheta}^{\mid}}|\xi|^{2}+\frac{3 \sigma_{a}}{4 a \bar{\vartheta}^{4}}
\end{array}\right) .
$$

Solving this equation with initial condition $\widehat{U}_{0}(\xi)$ we get

$$
\widehat{U}(t, \xi)=\widetilde{A}_{0}^{-1} \exp [t E(\xi)] \widehat{U}_{0}(\xi) .
$$


In the strictly hyperbolic case $\widetilde{D}=0$, under the Kalman rank condition [20] for the pair $(A(\xi), B)$, it can be proved [3] that

$$
\exists C>0, \quad \lambda(\xi)>0 \quad: \quad \exp [t E(\xi)] \leq C e^{-\lambda(\xi) t} .
$$

Observing the partially parabolic character of the system, one can expect a similar result when $\widetilde{D} \neq 0$ with a parabolic smoothing effect at low frequencies and an extra damping in the high frequency regime.

Taking benefit of the damping, we can use the Shizuta-Kawashima condition (SK) [32] which applies to the previous systems when $\alpha>0$. Following the arguments of Beauchard and Zuazua [3], we have

Lemma 1. For any $\xi \in S^{2}$, the matrices $B(\xi)$ and $A(\xi)$ satisfy the Shizuta-Kawashima condition $(S K)$ :

$$
\left\{\text { eigenvectors of }\left(\widetilde{A}_{0}\right)^{-1} A(\xi)\right\} \cap \operatorname{Ker} B(\xi)=\{0\} \text {. }
$$

As suggested in [3], one expects that the previous systems are strongly stable in $L^{2}$ : all $L^{2}$ solutions tend to zero in $L^{2}$ when $t \rightarrow \infty$.

Proof: One first checks that $\operatorname{Ker} B(\xi)$ is the 1-dimensional subspace spanned by the vector $(1,0,0,0,0,0)$. Therefore, if $X \in \operatorname{Ker} B(\xi) \backslash\{0\}$ is an eigenvector of $\left(\widetilde{A}_{0}\right)^{-1} A(\xi)$, we have $X=\left(x_{1}, 0,0,0,0,0\right), x_{1} \neq 0$, and

$$
A(\xi) X=\lambda \widetilde{A}_{0} X,
$$

for some $\lambda \in \mathbb{R}$. According to the values of $\widetilde{A}_{0}$ and $A(\xi)$, this implies that $\lambda=0, \xi_{1}=\xi_{2}=\xi_{3}=0$, which is in contradiction with the hypotesis $\xi \in S^{2}$.

Remark 4.1. It is clear in the above proof that $\nu>0$ is necessary. Indeed, in the case $\nu=0$, one easily sees that (4.20) is not satisfied. Indeed, in such a case, any vector of the form $X=$ $\left(0, \omega_{1}, \omega_{2}, \omega_{3}, 0,0\right)^{T}$ satisfies $A(\xi) X=0$ and $B(\xi) X=0$.

The stability condition (4.20) was first introduced in [32]. It was also used in [24] and [3] to prove global existence for hyperbolic-parabolic systems. It was proved in [32] that (4.20) is equivalent to the existence of a compensating matrix:

Proposition 4.1. There exists a matrix-valued function

$$
\begin{aligned}
K: \quad S^{2} & \longrightarrow \mathbb{R}^{6 \times 6} \\
\omega & \longmapsto K(\omega)
\end{aligned}
$$

such that

1. $\omega \mapsto K(\omega)$ is a $C^{\infty}$ function, and satisfies $K(-\omega)=-K(\omega)$ for any $\omega \in S^{2}$.

2. $K(\omega) \tilde{A}_{0}$ is a skew-symmetric matrix for any $\omega \in S^{2}$.

3. Denoting by $[A]=\frac{1}{2}\left(A+A^{T}\right)$ the symmetric part of $A$, the matrix $[K(\omega) A(\omega)]+B(\omega)$ is symmetric positive definite for any $\omega \in S^{2}$.

The proof of the equivalence between the Shizuta-Kawashima stability condition and the existence of a compensating matrix (Proposition 4.1) can be found in [21]. As in [24] and [3], we will use the existence of the compensating matrix $K(\omega)$ as a fundamental property allowing to prove global existence. 


\section{Entropy properties}

Adding equations (1.6) and (1.7) we get

$$
\partial_{t}\left(\frac{1}{2} \varrho|\vec{u}|^{2}+\varrho e+E_{r}\right)+\operatorname{div}_{x}\left(\left(\varrho E+E_{r}\right) \vec{u}+\left(p+p_{r}\right) \vec{u}\right)=\operatorname{div}_{x}\left(\kappa \nabla_{x} \vartheta\right)+\operatorname{div}_{x}\left(\frac{1}{3 \sigma_{s}} \nabla_{x} E_{r}\right) .
$$

Introducing the entropy $s$ of the fluid by the Gibbs law $\vartheta d s=d e+p d\left(\frac{1}{\varrho}\right)$ and denoting by $S_{r}:=\frac{4}{3} a T_{r}^{3}$ the radiative entropy, equation (1.7) rewrites

$$
\partial_{t} S_{r}+\operatorname{div}_{x}\left(S_{r} \vec{u}\right)=\frac{1}{T_{r}} \operatorname{div}_{x}\left(\frac{1}{3 \sigma_{s}} \nabla_{x} E_{r}\right)-\sigma_{a} \frac{E_{r}-a \vartheta^{4}}{T_{r}}
$$

or

$$
\partial_{t} S_{r}+\operatorname{div}_{x}\left(S_{r} \vec{u}\right)=\operatorname{div}_{x}\left(\frac{1}{3 \sigma_{s} T_{r}} \nabla_{x} E_{r}\right)+\frac{4 a}{3 \sigma_{s}} T_{r}\left|\nabla_{x} T_{r}\right|^{2}-\sigma_{a} \frac{E_{r}-a \vartheta^{4}}{T_{r}}
$$

Replacing equation (1.6) by the internal energy equation

$$
\partial_{t}(\varrho e)+\operatorname{div}_{x}(\varrho e \vec{u})+p \operatorname{div}_{x} \vec{u}-\nu|\vec{u}|^{2}=\operatorname{div}_{x}\left(\kappa \nabla_{x} \vartheta\right)-\sigma_{a}\left(a \vartheta^{4}-E_{r}\right),
$$

and dividing it by $\vartheta$, we may write an entropy equation for matter

$$
\partial_{t}(\varrho s)+\operatorname{div}_{x}(\varrho s \vec{u})-\frac{\nu}{\vartheta}|\vec{u}|^{2}=\operatorname{div}_{x}\left(\frac{\kappa \nabla_{x} \vartheta}{\vartheta}\right)+\frac{\kappa\left|\nabla_{x} \vartheta\right|^{2}}{\vartheta^{2}}-\sigma_{a} \frac{a \vartheta^{4}-E_{r}}{\vartheta} .
$$

So adding (5.4) and (5.2) we obtain

$$
\begin{gathered}
\partial_{t}\left(\varrho s+S_{r}\right)+\operatorname{div}_{x}\left(\left(\varrho s+S_{r}\right) \vec{u}\right)-\operatorname{div}_{x}\left(\frac{\kappa \nabla_{x} \vartheta}{\vartheta}+\frac{1}{3 \sigma_{s} T_{r}} \nabla_{x} E_{r}\right) \\
=\frac{\kappa\left|\nabla_{x} \vartheta\right|^{2}}{\vartheta^{2}}+\frac{4 a}{3 \sigma_{s}} T_{r}\left|\nabla_{x} E_{r}\right|^{2}+\frac{a \sigma_{a}}{\vartheta T_{r}}\left(\vartheta-T_{r}\right)^{2}\left(\vartheta+T_{r}\right)\left(\vartheta^{2}+T_{r}^{2}\right)+\frac{\nu}{\vartheta}|\vec{u}|^{2} .
\end{gathered}
$$

Introducing the Helmholtz functions $H_{\bar{\vartheta}}(\varrho, \vartheta):=\varrho(e-\bar{\vartheta} s)$ and $H_{r, \bar{\vartheta}}\left(T_{r}\right):=E_{r}-\bar{\vartheta} S_{r}$, we check that the quantities $H_{\bar{\vartheta}}(\varrho, \vartheta)-(\varrho-\bar{\varrho}) \partial_{\varrho} H_{\bar{\vartheta}}(\bar{\varrho}, \bar{\vartheta})-H_{\bar{\vartheta}}(\bar{\varrho}, \bar{\vartheta})$ and $H_{r, \bar{\vartheta}}\left(T_{r}\right)-H_{r, \bar{\vartheta}}\left(\bar{T}_{r}\right)$ are non-negative and strictly coercive functions reaching zero minima at the equilibrium state $\left(\bar{\varrho}, \bar{\vartheta}, \bar{E}_{r}\right)$.

Lemma 2. Let $\bar{\varrho}, \bar{\vartheta}$ and $\bar{T}_{r}$ be three given positive constants. Let $\mathcal{O}_{1}$ and $\mathcal{O}_{2}$ be the sets defined by

$$
\begin{gathered}
\mathcal{O}_{1}:=\left\{(\varrho, \vartheta) \in \mathbb{R}^{2}: \frac{\bar{\varrho}}{2}<\varrho<2 \bar{\varrho}, \frac{\bar{\vartheta}}{2}<\vartheta<2 \bar{\vartheta},\right\} . \\
\mathcal{O}_{2}:=\left\{T_{r} \in \mathbb{R}: \frac{\bar{T}_{r}}{2}<T_{r}<2 \bar{T}_{r},\right\} .
\end{gathered}
$$

There exist positive constants $C_{1,2}(\bar{\varrho}, \bar{\vartheta})$ and $C_{3,4}\left(\bar{T}_{r}\right)$ such that

1.

$$
\begin{gathered}
C_{1}\left(|\varrho-\bar{\varrho}|^{2}+|\vartheta-\bar{\vartheta}|^{2}\right) \leq H_{\bar{\vartheta}}(\varrho, \vartheta)-(\varrho-\bar{\varrho}) \partial_{\varrho} H_{\bar{\vartheta}}(\bar{\varrho}, \bar{\vartheta})-H_{\bar{\vartheta}}(\bar{\varrho}, \bar{\vartheta}) \\
\leq C_{2}\left(|\varrho-\bar{\varrho}|^{2}+|\vartheta-\bar{\vartheta}|^{2}\right)
\end{gathered}
$$

for all $(\varrho, \vartheta) \in \mathcal{O}_{1}$, 
2.

$$
C_{3}\left|T_{r}-\bar{T}_{r}\right|^{2} \leq H_{r, \bar{\vartheta}}\left(T_{r}\right)-H_{r, \bar{\vartheta}}\left(\bar{T}_{r}\right) \leq C_{4}\left|T_{r}-\bar{T}_{r}\right|^{2}
$$

for all $T_{r} \in \mathcal{O}_{2}$.

\section{Proof:}

1. Point 1 is proved in [12] and we only sketch the proof for convenience.

According to the decomposition

$$
\varrho \rightarrow H_{\bar{\vartheta}}(\varrho, \vartheta)-(\varrho-\bar{\varrho}) \partial_{\varrho} H_{\bar{\vartheta}}(\bar{\varrho}, \bar{\vartheta})-H_{\bar{\vartheta}}(\bar{\varrho}, \bar{\vartheta})=\mathcal{F}(\varrho)+\mathcal{G}(\varrho),
$$

where $\mathcal{F}(\varrho)=H_{\bar{\vartheta}}(\varrho, \bar{\vartheta})-(\varrho-\bar{\varrho}) \partial_{\varrho} H_{\bar{\vartheta}}(\bar{\varrho}, \bar{\vartheta})-H_{\bar{\vartheta}}(\bar{\varrho}, \bar{\vartheta})$ and $\mathcal{G}(\varrho)=H_{\bar{\vartheta}}(\varrho, \vartheta)-H_{\bar{\vartheta}}(\varrho, \bar{\vartheta})$, one checks that $\mathcal{F}$ is strictly convex and reaches a zero minimum at $\bar{\varrho}$, while $\mathcal{G}$ is strictly decreasing for $\vartheta<\bar{\vartheta}$ and strictly increasing for $\vartheta>\bar{\vartheta}$, after thermodynamic stability properties (2.2) and (2.3). Computing the derivatives of $H_{\bar{\vartheta}}$ leads directly to the estimate (5.8).

2. Point 2 follows immediately from the properties of the function $x \rightarrow H_{r, \bar{\vartheta}}(x)-H_{r, \bar{\vartheta}}\left(T_{r}\right)=$ $a x^{3}\left(x-\frac{4}{3} \bar{\vartheta}\right)+\frac{a}{3} \bar{\vartheta}^{4}$.

From this simple result, we can obtain an identity leading to energy estimates. In fact, subtracting (5.5) from (5.1) and using the conservation of mass, we get

$$
\begin{array}{r}
\partial_{t}\left(\frac{1}{2} \varrho|\vec{u}|^{2}+H_{\bar{\vartheta}}(\varrho, \vartheta)-(\varrho-\bar{\varrho}) \partial_{\varrho} H_{\bar{\vartheta}}(\bar{\varrho}, \bar{\vartheta})-H_{\bar{\vartheta}}(\bar{\varrho}, \bar{\vartheta})+H_{r, \bar{\vartheta}}\left(T_{r}\right)\right) \\
=\operatorname{div}_{x}\left(\left(\varrho E+E_{r}\right) \vec{u}+\left(p+p_{r}\right) \vec{u}+\bar{\vartheta}\left(\varrho s+S_{r}\right) \vec{u}\right) \\
+\operatorname{div}_{x}\left(\kappa \nabla_{x} \vartheta+\frac{1}{3 \sigma_{s}} \nabla_{x} E_{r}\right)+\bar{\vartheta} \operatorname{div}_{x}\left(\frac{\kappa \nabla_{x} \vartheta}{\vartheta}+\frac{1}{3 \sigma_{s} T_{r}} \nabla_{x} E_{r}\right) \\
-\bar{\vartheta} \frac{\kappa\left|\nabla_{x} \vartheta\right|^{2}}{\vartheta^{2}}-\bar{\vartheta} \frac{4 a}{3 \sigma_{s}} T_{r}\left|\nabla_{x} E_{r}\right|^{2}-\bar{\vartheta} \frac{a \sigma_{a}}{\vartheta T_{r}}\left(\vartheta-T_{r}\right)^{2}\left(\vartheta+T_{r}\right)\left(\vartheta^{2}+T_{r}^{2}\right)-\frac{\nu}{\vartheta}|\vec{u}|^{2} .
\end{array}
$$

In the sequel, we define $V=\left(\rho, \vec{u}, \vartheta, E_{r}\right)^{T}, \bar{V}=\left(\bar{\rho}, 0, \bar{\vartheta}, \overline{E_{r}}\right)^{T}$, and

$$
\begin{aligned}
N(t)^{2}=\sup _{0 \leq s \leq t} & \|V(s)-\bar{V}\|_{H^{d}\left(\mathbb{R}^{3}\right)}^{2} \\
& +\int_{0}^{t}\left(\left\|\nabla_{x} V(s)\right\|_{H^{d-1}}^{2}\left(\mathbb{R}^{3}\right)+\left\|\nabla_{x} \vartheta(s)\right\|_{H^{d}\left(\mathbb{R}^{3}\right)}^{2}+\left\|\nabla_{x} E_{r}(s)\right\|_{H^{d}\left(\mathbb{R}^{3}\right)}^{2}\right) d s \\
& \quad+\int_{0}^{t}\left(\left\|\vartheta(s)-T_{r}(s)\right\|_{H^{d-1}\left(\mathbb{R}^{3}\right)}+\|\vec{u}\|_{H^{d-1}\left(\mathbb{R}^{3}\right)}\right) d s .
\end{aligned}
$$

Recall that $T_{r}=E_{r}^{1 / 4} a^{-1 / 4}$.

\section{$5.1 \quad L^{\infty}\left(H^{d}\right)$ estimates}

Using these entropy properties, we are going to prove the following result: 
Proposition 5.1. Let the assumptions of Theorem 3.2 be satisfied. Consider a solution $\left(\varrho, \vec{u}, \vartheta, E_{r}\right)$ of system (1.4)-(1.5)-(1.6) on $[0, t]$, for some $t>0$. Then, the energy defined by (5.11) satisfies

$$
\begin{array}{r}
\|V(t)-\bar{V}\|_{L^{2}\left(\mathbb{R}^{3}\right)}^{2}+\int_{0}^{t}\left(\left\|\nabla_{x} \vartheta(s)\right\|_{L^{2}\left(\mathbb{R}^{3}\right)}^{2}+\left\|\nabla_{x} E_{r}(s)\right\|_{L^{2}\left(\mathbb{R}^{3}\right)}^{2}+\left\|\vartheta-T_{r}\right\|_{L^{2}\left(\mathbb{R}^{3}\right)}^{2}\right) d s \\
\leq C(N(t)) N(0)^{2}
\end{array}
$$

where the function $C$ is non-decreasing.

Proof: We follow the proof of [24, Lemma 3.1]: we define

$$
\eta(t, x)=H_{\bar{\vartheta}}(\varrho, \vartheta)-(\varrho-\bar{\varrho}) \partial_{\varrho} H_{\bar{\vartheta}}(\bar{\varrho}, \bar{\vartheta})-H_{\bar{\vartheta}}(\bar{\varrho}, \bar{\vartheta})+H_{r, \bar{\vartheta}}\left(T_{r}\right) .
$$

We multiply (5.5) by $\bar{\vartheta}$, and subtract the result to (5.1). Integrating over $[0, t] \times \mathbb{R}^{3}$, we find

$$
\begin{aligned}
\int_{\mathbb{R}^{3}}\left(\frac{1}{2} \varrho(t)|\vec{u}|^{2}(t)+\eta(t, x)\right) d x+\int_{0}^{t} \int_{\mathbb{R}^{3}} \kappa \frac{\bar{\vartheta}}{\vartheta^{2}}\left|\nabla_{x} \vartheta\right|^{2}+\frac{4 a}{3 \sigma_{s}} T_{r}\left|\nabla_{x} E_{r}\right|^{2} \bar{\vartheta} \\
+\int_{0}^{t} \int_{\mathbb{R}^{3}} \bar{\vartheta} \frac{a \sigma_{a}}{\vartheta T_{r}}\left(\vartheta+T_{r}\right)\left(\vartheta-T_{r}\right)^{2}\left(\vartheta^{2}+T_{r}^{2}\right)+\int_{0}^{t} \int_{\mathbb{R}^{3}} \overline{\bar{\vartheta}} \nu|\vec{u}|^{2} \leq \int_{\mathbb{R}^{3}} \eta(0, x) d x .
\end{aligned}
$$

Defining

$$
M(t)=\sup _{0 \leq s \leq t} \sup _{x \in \mathbb{R}^{3}}\left(\max \left(|\varrho(s, x)-\bar{\varrho}|,|\vec{u}(s, x)|,|\vartheta(s, x)-\bar{\vartheta}|, \mid E_{r}(s, x)-\overline{E_{r}}\right)\right),
$$

and applying Lemma 2, we find that

$$
\begin{array}{r}
\|V(t)-\bar{V}\|_{L^{2}\left(\mathbb{R}^{3}\right)}^{2}+\int_{0}^{t}\left(\left.\left|\nabla_{x} \vartheta(s)\left\|_{L^{2}\left(\mathbb{R}^{3}\right)}^{2}+\right\| \nabla_{x} E_{r}(s)\left\|_{L^{2}\left(\mathbb{R}^{3}\right)}^{2}+\right\| \vartheta-T_{r} \|_{L^{2}\left(\mathbb{R}^{3}\right)}^{2}+\frac{\nu \bar{\vartheta}}{\vartheta}\right| \vec{u}\right|^{2}\right) d s \\
\leq C(M(t)) N(0),
\end{array}
$$

where $C: \mathbb{R}^{+} \rightarrow \mathbb{R}^{+}$is non-decreasing. Finally, we point out that, since $d>7 / 2>3 / 2$, due to Sobolev embeddings, there exists a universal constante $C_{0}$ such that $M(t) \leq C_{0} N(t)$. Since $C$ is non-decreasing, this proves (5.12).

Proposition 5.2. Under the same assumptions as in Theorem 3.2, we have the following estimate: (here, we set $\left.V=\left(\varrho, \vec{u}, \vartheta, E_{r}\right)^{T}\right)$

$$
\begin{aligned}
\left\|\partial_{t} V(t)\right\|_{H^{d-1}\left(\mathbb{R}^{3}\right)} \leq C(N(t))\left(\left\|\nabla_{x} V\right\|_{H^{d-1}\left(\mathbb{R}^{3}\right)}\right. & +\left\|\nabla_{x} \vartheta\right\|_{H^{d}}\left(\mathbb{R}^{3}\right) \\
& +\left\|\nabla_{x} E_{r}\right\|_{H^{d}}\left(\mathbb{R}^{3}\right) \\
& \left.+\left\|\vartheta-T_{r}\right\|_{H^{d-1}\left(\mathbb{R}^{3}\right)}\right)
\end{aligned}
$$

Proof: The system satisfied by $V$ may be written formally

$$
A_{0}(V) \partial_{t} V+\sum_{j=1}^{3} A_{j}(V) \partial_{x_{j}} V=D(V) \Delta V-B(V) V .
$$

Therefore,

$$
\begin{array}{r}
\partial_{t} V=-\sum_{j=1}^{3}\left[\widetilde{A}_{j}(V)-\widetilde{A}_{j}(\bar{V})\right] \partial_{x_{j}} V-\sum_{j=1}^{n} \widetilde{A}_{j}(\bar{V}) \partial_{x_{j}} V+[\widetilde{D}(V)-\widetilde{D}(\bar{V})] \Delta V+\widetilde{D}(\bar{V}) \Delta V \\
-[\widetilde{B}(V)-\widetilde{B}(\bar{V})] V-\widetilde{B}(\bar{V}) V
\end{array}
$$


where $\widetilde{A}_{j}(V)=\left(A_{0}(V)\right)^{-1} A_{j}(V), \widetilde{B}(V)=\left(A_{0}(V)\right)^{-1} B(V)$, and $\widetilde{D}(V)=\left(A_{0}(V)\right)^{-1} D(V)$. We point out two important facts: First, these matrices are Lipschitz continuous with respect to $V$, away from $\varrho=0$ and $\vartheta=0$. Second, the matrices $\widetilde{B}$ and $\widetilde{D}$ have, respectively, the same structure as those defined in (4.15). Note also that, since $d-1>5 / 2=3 / 2+1$, Sobolev embeddings imply that $H^{d-1}\left(\mathbb{R}^{3}\right)$ is an algebra. Therefore, we, have

$$
\begin{aligned}
&\left\|\partial_{t} V\right\|_{H^{d-1}}\left(\mathbb{R}^{3}\right) \leq C_{0}\left(1+\sum_{j=1}^{3}\left\|\widetilde{A}_{j}(V)-\widetilde{A}_{j}(\bar{V})\right\|_{H^{d-1}\left(\mathbb{R}^{3}\right)}\right)\left\|\nabla_{x} V\right\|_{H^{d-1}\left(\mathbb{R}^{3}\right)} \\
&+C_{0}\left(1+\|\widetilde{D}(V)-\widetilde{D}(\bar{V})\|_{H^{d-1}\left(\mathbb{R}^{3}\right)}\right)\left(\|\Delta \vartheta\|_{H^{d-1}\left(\mathbb{R}^{3}\right)}+\left\|\Delta E_{r}\right\|_{H^{d-1}\left(\mathbb{R}^{3}\right)}\right) \\
&+C_{0}\left(1+\|\widetilde{B}(V)-\widetilde{B}(\bar{V})\|_{H^{d-1}\left(\mathbb{R}^{3}\right)}\right)\left\|\vartheta-T_{r}\right\|_{H^{d-1}\left(\mathbb{R}^{3}\right)},
\end{aligned}
$$

whence,

$$
\begin{aligned}
\left\|\partial_{t} V\right\|_{H^{d-1}}\left(\mathbb{R}^{3}\right) & \leq C_{0}\left(1+\|V-\bar{V}\|_{H^{d-1}\left(\mathbb{R}^{3}\right)}\right) \\
& \times\left(\left\|\nabla_{x} V\right\|_{H^{d-1}\left(\mathbb{R}^{3}\right)}+\|\Delta \vartheta\|_{H^{d-1}\left(\mathbb{R}^{3}\right)}+\left\|\Delta E_{r}\right\|_{H^{d-1}\left(\mathbb{R}^{3}\right)}+\left\|\vartheta-T_{r}\right\|_{H^{d-1}\left(\mathbb{R}^{3}\right)}\right),
\end{aligned}
$$

which proves (5.15).

Next, we bound the spatial derivatives as follows:

Proposition 5.3. Assume that the hypotheses of Theorem 3.2 are satisfied. Let $k \in \mathbb{N}^{3}$ be such that $1 \leq|k| \leq d$, where $d>7 / 2$. Then, we have

$$
\begin{gathered}
\left\|\partial_{x}^{k} V(t)\right\|_{L^{2}\left(\mathbb{R}^{3}\right)}+\int_{0}^{t}\left(\left\|\partial_{x}^{k} \nabla_{x} \vartheta(s)\right\|_{L^{2}\left(\mathbb{R}^{3}\right)}^{2}+\left\|\partial_{x}^{k} \nabla_{x} E_{r}(s)\right\|_{L^{2}\left(\mathbb{R}^{3}\right)}^{2}+\left\|\partial_{x}^{k}\left(\vartheta-T_{r}\right)\right\|_{L^{2}\left(\mathbb{R}^{3}\right)}\right) d s \\
\leq C_{0} N(0)^{2} \\
+C_{0} N(t) \int_{0}^{t}\left(\left\|\nabla_{x} V(s)\right\|_{H^{d-1}\left(\mathbb{R}^{3}\right)}^{2}+\left\|\nabla_{x} \vartheta(s)\right\|_{H^{d}\left(\mathbb{R}^{3}\right)}+\left\|\nabla_{x} E_{r}\right\|_{H^{d}\left(\mathbb{R}^{3}\right)}+\left\|\vartheta-T_{r}\right\|_{H^{d-1}\left(\mathbb{R}^{3}\right)}\right) d s
\end{gathered}
$$

Proof: We write the system (1.4)-(1.5)-(1.6) as

$$
A_{0}(V) \partial_{t} V+\sum_{j=1}^{3} A_{j}(V) \partial_{x_{j}} V=D \Delta V-B(V) V .
$$

We apply $\partial_{x}^{k}$ to this system, then take the scalar product with the vector $\partial_{x}^{k} V$, and integrate over $[0, t] \times \mathbb{R}^{3}$. We find

$$
\begin{array}{r}
\frac{1}{2} \int_{\mathbb{R}^{3}}\left[\left(A_{0} \partial_{x}^{k} V\right) \cdot \partial_{x}^{k} V\right]_{0}^{t}+\int_{0}^{t} \int_{\mathbb{R}^{3}}\left(D \nabla_{x}\left(\partial_{x}^{k} V\right)\right) \cdot \nabla_{x}\left(\partial_{x}^{k} V\right)+\int_{0}^{t} \int_{\mathbb{R}^{3}}\left(B\left(\partial_{x}^{k} V\right)\right) \cdot\left(\partial_{x}^{k} V\right) \\
=\int_{0}^{t} \int_{\mathbb{R}^{3}}\left(\frac{1}{2}\left(I_{1}+I_{2}\right)-I_{3}-I_{4}+I_{5}\right),
\end{array}
$$


where

$$
\begin{array}{ll}
I_{1}=\partial_{t}\left(A_{0}(v)\right) \partial_{x}^{k} V \cdot \partial_{x}^{k} V, & I_{2}=\sum_{j=1}^{3} \partial_{x_{j}}\left(A_{j}(V)\right) \partial_{x}^{k} V \cdot \partial_{x}^{k} V, \quad I_{3}=\left[\partial_{x}^{k}, A_{0}(V)\right] \partial_{t} V \cdot \partial_{x}^{k} V, \\
I_{4}=\sum_{j=1}^{3}\left[\partial_{x}^{k}, A_{j}(V)\right] \partial_{x_{j}} V \cdot \partial_{x}^{k} V, & I_{5}=\partial_{x}^{k}(B(V)) V \cdot \partial_{x}^{k} V .
\end{array}
$$

We estimate separately each term of the right-hand side. First, we have

$$
\begin{aligned}
\int_{0}^{t} \int_{\mathbb{R}^{3}}\left|I_{1}\right| & \leq C \int_{0}^{t} \int_{\mathbb{R}^{3}}\left|\partial_{x}^{k} V\right|^{2}\left|\partial_{t} V\right| \\
& \leq C \int_{0}^{t} \int_{\mathbb{R}^{3}}\left|\partial_{x}^{k} V\right|^{2}\left(\left|\nabla_{x} V\right|+|B(V) V|+|D \Delta V|\right) \\
& \leq C N(t) \int_{0}^{t}\left\|\partial_{x}^{k} V(s)\right\|_{L^{2}\left(\mathbb{R}^{3}\right)}^{2} d s,
\end{aligned}
$$

where we have used Sobolev embeddings and the fact that $d>7 / 2$. A similar computation gives

$$
\int_{0}^{t} \int_{\mathbb{R}^{3}}\left|I_{2}\right| \leq C N(t) \int_{0}^{t}\left\|\partial_{x}^{k} V(s)\right\|_{L^{2}\left(\mathbb{R}^{3}\right)}^{2} d s .
$$

We estimate $I_{3}$ by applying Cauchy-Schwarz inequality:

$$
\int_{0}^{t} \int_{\mathbb{R}^{3}}\left|I_{3}\right| \leq \int_{0}^{t}\left\|\partial_{x}^{k} V\right\|_{L^{2}\left(\mathbb{R}^{3}\right)}\left\|\left[\partial_{x}^{k}, A_{0}(V)\right] \partial_{t} V\right\|_{L^{2}\left(\mathbb{R}^{3}\right)}
$$

Then, we apply same estimate for commutators and composition of functions (see [26, Proposition $2.1])$, and $|k| \leq d$ :

$$
\begin{aligned}
& \left\|\left[\partial_{x}^{k}, A_{0}(V)\right] \partial_{t} V\right\|_{L^{2}\left(\mathbb{R}^{3}\right)}=\left\|\left[\partial_{x}^{k}, A_{0}(V)-A_{0}(\bar{V})\right] \partial_{t} V\right\|_{L^{2}\left(\mathbb{R}^{3}\right)} \\
& \leq C\left(\left\|\partial_{t} V\right\|_{L^{\infty}\left(\mathbb{R}^{3}\right)}\left\|\nabla_{x} A_{0}(V)\right\|_{H^{d-1}\left(\mathbb{R}^{3}\right)}+\left\|\partial_{t} V\right\|_{H^{d-1}\left(\mathbb{R}^{3}\right)}\left\|\nabla_{x} A_{0}(V)\right\|_{L^{\infty}\left(\mathbb{R}^{3}\right)}\right) .
\end{aligned}
$$

Moreover, we have

$$
\left\|\nabla_{x} A_{0}(V)\right\|_{H^{d-1}\left(\mathbb{R}^{3}\right)} \leq C\|V-\bar{V}\|_{H^{d}\left(\mathbb{R}^{3}\right)} \leq C N(t),
$$

and

Hence, $I_{3}$ satisfies

$$
\left\|\nabla_{x} A_{0}(V)\right\|_{L^{\infty}\left(\mathbb{R}^{3}\right)} \leq C\left\|\nabla_{x} V\right\|_{H^{d-1}\left(\mathbb{R}^{3}\right)} \leq C N(t)
$$

$$
\int_{0}^{t} \int_{\mathbb{R}^{3}}\left|I_{3}\right| \leq C N(t) \int_{0}^{t}\left(\left\|\nabla_{x} V(s)\right\|_{H^{d}}^{2}\left(\mathbb{R}^{3}\right)\right) d s
$$

Here, we have used (5.15). The integral of $I_{4}$ is dealt with using similar computations. Turning to $I_{5}$, we use the particular form of $\partial_{x}^{k} B(V)$, which has non-zero entries only on its last two lines and its fifth column. More precisely, we have

$$
\partial_{x}^{k}(B(V)) V=\left(\begin{array}{c}
0 \\
0 \\
0 \\
0 \\
a \sigma_{a} \partial_{x}^{k}\left(\vartheta^{3}\right) \\
-a \sigma_{a} \partial_{x}^{k}\left(\vartheta^{3}\right)
\end{array}\right),
$$


hence

$$
\partial_{x}^{k}(B(V)) V \cdot \partial_{x}^{k} V=a \sigma_{a} \partial_{x}^{k}\left(\vartheta^{3}\right) \partial_{x}^{k}\left(\vartheta-E_{r}\right)
$$

from which we infer

$$
\int_{0}^{t} \int_{\mathbb{R}^{3}}\left|I_{5}\right| \leq C N(t) \int_{0}^{t}\left\|\partial_{x}^{k} V(s)\right\|_{L^{2}\left(\mathbb{R}^{3}\right)}^{2} d s
$$

Collecting the estimates on $I_{1}, I_{2}, I_{3}, I_{4}$ and $I_{5}$, we prove (5.16).

The above results allow to derive the following bound:

Proposition 5.4. Assume that the assumptions of Proposition 5.1 are satisfied. Then, there exists a non-decreasing function $C: \mathbb{R}^{+} \rightarrow \mathbb{R}^{+}$such that

$$
\begin{array}{r}
\|V-\bar{V}\|_{H^{d}\left(\mathbb{R}^{3}\right)}^{2}+\int_{0}^{t}\left(\left\|\nabla_{x} \vartheta(s)\right\|_{H^{d}\left(\mathbb{R}^{3}\right)}^{2}+\left\|\nabla_{x} E_{r}(s)\right\|_{H^{d}}^{2}\left(\mathbb{R}^{3}\right)+\left\|\vartheta-T_{r}\right\|_{H^{d}\left(\mathbb{R}^{3}\right)}^{2}\right) d s \\
\leq C(N(t))\left(N(0)^{2}+N(t) \int_{0}^{t}\left\|\nabla_{x} \vartheta(s)\right\|_{H^{d-1}\left(\mathbb{R}^{3}\right)}^{2} d s\right) .
\end{array}
$$

Proof: We sum up estimates (5.16) over all multi-indices $k$ such that $|k| \leq d$, and add this to (5.15). This leads to (5.17).

\section{$5.2 \quad L^{2}\left(H^{d-1}\right)$ estimates}

In this section, we derive bounds on the right-hand side of (5.17). For this purpose, we adapt the strategy of [32], which was further developed in [15]. We apply the Fourier transform to the linearized system and use the compensating matrix $K$ to prove estimates on the space derivatives of $V$.

Proposition 5.5. Assume that the assumptions of Proposition 5.1 are satisfied. Then there exists a non-decreasing function $C: \mathbb{R}^{+} \rightarrow \mathbb{R}^{+}$such that

$$
\int_{0}^{t}\left\|\nabla_{x} V(s)\right\|_{H^{d-1}\left(\mathbb{R}^{3}\right)} d s \leq C(N(t))\left(N(t)+\left\|V_{0}-\bar{V}\right\|_{H^{d}}\left(\mathbb{R}^{3}\right)\right)
$$

Proof: As a first step, we apply the symmetrizer of the linearized system (4.13) (which leads to (4.14)) to the nonlinear system (1.4)-(1.5)-(1.6), which then reads

$$
\widetilde{A}_{0}(V) \partial_{t} V+\sum_{j=1}^{3} \widetilde{A}_{j}(V) \partial_{x_{j}} V=\widetilde{D} \Delta V-\widetilde{B}(V) V
$$

Of course, this system is not symmetric. However, the corresponding linearized system (4.14) is symmetric. Next, we rewrite the nonlinear system by setting $U=V-\bar{V}$ :

$$
\widetilde{A}_{0}(V) \partial_{t} U+\sum_{j=1}^{3} \widetilde{A}_{j}(V) \partial_{x_{j}} U=\widetilde{D} \Delta U-\widetilde{B}(V) U-\widetilde{B}(V) \bar{V}
$$

Therefore, multiplying this system on the left by $\widetilde{A}_{0}(\bar{V})\left(\widetilde{A}_{0}(V)\right)^{-1}$, we find

$$
\widetilde{A}_{0}(\bar{V}) \partial_{t} U+\sum_{j=1}^{3} \widetilde{A}_{j}(\bar{V}) \partial_{x_{j}} U=H
$$


where

$$
\begin{aligned}
H=- & \widetilde{A}_{0}(\bar{V}) \sum_{j=1}^{3}\left[\left(\widetilde{A}_{0}(V)\right)^{-1} \widetilde{A}_{j}(V)-\left(\widetilde{A}_{0}(\bar{V})\right)^{-1} \widetilde{A}_{j}(\bar{V})\right] \partial_{x_{j}} V \\
& +\widetilde{A}_{0}(\bar{V})\left(\widetilde{A}_{0}(V)\right)^{-1} \widetilde{D} \Delta U-\widetilde{A}_{0}(\bar{V})\left(\widetilde{A}_{0}(V)\right)^{-1} \widetilde{B}(V) U-\widetilde{A}_{0}(\bar{V})\left(\widetilde{A}_{0}(V)\right)^{-1} \widetilde{B}(V) \bar{V} .
\end{aligned}
$$

We apply the Fourier transform to (5.19), and then multiply on the left by $-i(\widehat{U})^{*} K\left(\frac{\xi}{|\xi|}\right)$, where ${ }^{*}$ denotes the transpose of the complex conjugate, and $K$ is the compensating matrix (see Proposition 4.1). Taking the real part of the result, we infer

$$
\begin{aligned}
\operatorname{Im}\left((\widehat{U})^{*} K\left(\frac{\xi}{|\xi|}\right) A_{0}(\bar{V}) \partial_{t} \widehat{U}\right)+|\xi|(\widehat{U})^{*} K\left(\frac{\xi}{|\xi|}\right) A\left(\frac{\xi}{|\xi|}\right) \widehat{U} & \\
& =\operatorname{Im}\left((\widehat{U})^{*} K\left(\frac{\xi}{|\xi|}\right) \widehat{H}\right),
\end{aligned}
$$

where the matrix $A\left(\frac{\xi}{|\xi|}\right)$ is defined by (4.18). According to Proposition 4.1, $K A_{0}(\bar{V})$ is skewsymmetric, hence

$$
\operatorname{Im}\left((\widehat{U})^{*} K\left(\frac{\xi}{|\xi|}\right) A_{0}(\bar{V}) \partial_{t} \widehat{U}\right)=\frac{1}{2} \frac{d}{d t} \operatorname{Im}\left((\widehat{U})^{*} K\left(\frac{\xi}{|\xi|}\right) A_{0}(\bar{V}) \widehat{U}\right) .
$$

Next, we also have

$$
\begin{aligned}
|\xi|(\widehat{U})^{*} K\left(\frac{\xi}{|\xi|}\right) A\left(\frac{\xi}{|\xi|}\right) \widehat{U}=|\xi|(\widehat{U})^{*}\left[K\left(\frac{\xi}{|\xi|}\right) A\left(\frac{\xi}{|\xi|}\right)+B\left(\frac{\xi}{|\xi|}\right)\right] \widehat{U} & \\
& -|\xi|(\widehat{U})^{*} \widetilde{B} \widehat{U}-|\xi|(\widehat{U})^{*} \widetilde{D} \widehat{U} .
\end{aligned}
$$

Hence, still applying Proposition 4.1, there exists $\alpha_{1}>0$ and $\alpha_{2}>0$ such that

$$
\begin{aligned}
|\xi|(\widehat{U})^{*} K\left(\frac{\xi}{|\xi|}\right) A\left(\frac{\xi}{|\xi|}\right) \widehat{U} \geq & \alpha_{1}|\xi||\widehat{U}|^{2} \\
& \quad-\alpha_{2} \frac{1}{|\xi|}\left(|\xi(\widehat{\vartheta-\bar{\vartheta}})|^{2}+\left|\xi\left(\widehat{E_{r}-E_{r}}\right)\right|^{2}+|\xi|^{2}|\widehat{\vec{u}}|^{2}\right) .
\end{aligned}
$$

Finally, we estimate the right-hand side of (5.20) using Cauchy-Schwarz inequality and Young inequality:

$$
\left|\operatorname{Im}\left((\widehat{U})^{*} K\left(\frac{\xi}{|\xi|}\right) \widehat{H}\right)\right| \leq \varepsilon|\xi||\widehat{U}|^{2}+C_{\varepsilon} \frac{1}{|\xi|}|\widehat{H}|^{2}
$$

for any $\varepsilon>0$. We choose $\varepsilon$ small enough, insert (5.21)-(5.22)-(5.23) into (5.20), and find

$$
\begin{aligned}
|\xi||\widehat{U}|^{2} \leq C\left[\frac{1}{|\xi|}\left(|\xi(\widehat{\vartheta-\bar{\vartheta}})|^{2}+\left|\xi\left(\widehat{E_{r}-\overline{E_{r}}}\right)\right|^{2}+|\xi|^{2}|\widehat{\vec{u}}|^{2}\right)+\frac{1}{|\xi|}|\widehat{H}|^{2}\right. & \\
& \left.-\frac{d}{d t} \operatorname{Im}\left((\widehat{U})^{*} K\left(\frac{\xi}{|\xi|}\right) A_{0}(\bar{V}) \widehat{U}\right)\right] .
\end{aligned}
$$

We multiply this inequality by $|\xi|^{2 l-1}$, for some $1 \leq l \leq d$, and get

$$
\begin{array}{r}
|\xi|^{2 l}|\widehat{U}|^{2} \leq C\left[|\xi|^{2 l-2}\left(|\xi(\widehat{\vartheta-\bar{\vartheta}})|^{2}+\left|\xi\left(\widehat{E_{r}-E_{r}}\right)\right|^{2}+|\xi|^{2}|\widehat{\vec{u}}|^{2}\right)+|\xi|^{2 l-2}|\widehat{H}|^{2}\right. \\
\left.-|\xi|^{2 l-1} \frac{d}{d t} \operatorname{Im}\left((\widehat{U})^{*} K\left(\frac{\xi}{|\xi|}\right) A_{0}(\bar{V}) \widehat{U}\right)\right] .
\end{array}
$$




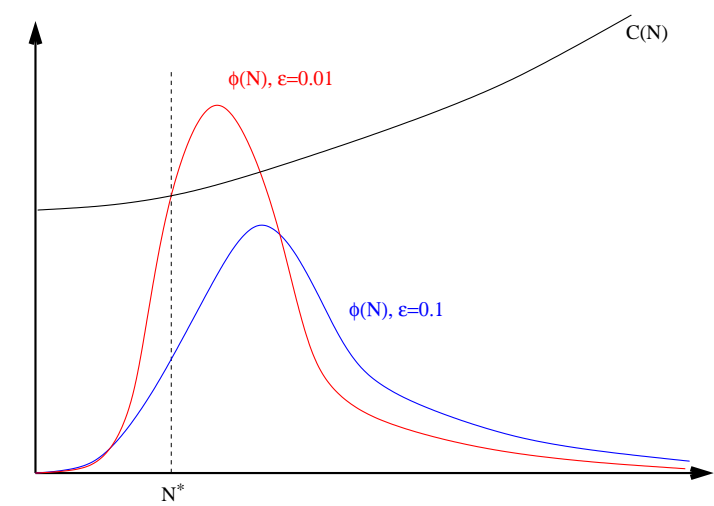

Figure 1: The functions $\phi$ and $C$ used in the proof of Theorem 3.2

We integrate this inequality over $[0, t] \times \mathbb{R}^{3}$, and use Plancherel's theorem:

$$
\begin{aligned}
\int_{0}^{t} \int_{\mathbb{R}^{3}} \sum_{|k|=l}\left|\partial_{x}^{k} \nabla_{x} V\right|^{2} \leq C \int_{0}^{t} \int_{\mathbb{R}^{3}} \sum_{|k|=l} & \left(\left|\partial_{x}^{k} \nabla_{x} \vartheta\right|^{2}+\left|\partial_{x}^{k} \nabla_{x} E_{r}\right|^{2}+\left|\partial_{x}^{k} \nabla_{x} \vec{u}\right|^{2}+\left|\partial_{x}^{k} H\right|^{2}\right) \\
& +C \operatorname{Im} \int_{\mathbb{R}^{3}}|\xi|^{2 l-1}\left[(\widehat{U})^{*} K\left(\frac{\xi}{|\xi|}\right) A_{0}(\bar{V}) \widehat{U}\right]_{0}^{t}
\end{aligned}
$$

The matrix $K\left(\frac{\xi}{|\xi|}\right)$ is uniformly bounded for $\xi \in \mathbb{R}^{3} \backslash\{0\}$, so we have

$$
\begin{array}{r}
\operatorname{Im} \int_{\mathbb{R}^{3}}|\xi|^{2 l-1}\left[(\widehat{U})^{*} K\left(\frac{\xi}{|\xi|}\right) A_{0}(\bar{V}) \widehat{U}\right]_{0}^{t} \leq C \\
\left(\int_{\mathbb{R}^{3}}\left(1+|\xi|^{2}\right)^{l}|\widehat{U}(t)|^{2}+\int_{\mathbb{R}^{3}}\left(1+|\xi|^{2}\right)^{l}\left|\widehat{U}_{0}\right|^{2}\right) \\
\leq C\left(\|V-\bar{V}\|_{H^{l}\left(\mathbb{R}^{3}\right)}+\left\|V_{0}-\bar{V}\right\|_{H^{l}\left(\mathbb{R}^{3}\right)}\right)
\end{array}
$$

We insert this estimate into (5.25), sum the result over $1 \leq l \leq d$, which leads to

$$
\begin{aligned}
& \int_{0}^{t}\left\|\nabla_{x} V\right\|_{H^{d-1}\left(\mathbb{R}^{3}\right)} \leq C\left(\|V-\bar{V}\|_{H^{d}\left(\mathbb{R}^{3}\right)}+\left\|V_{0}-\bar{V}\right\|_{H^{d}\left(\mathbb{R}^{3}\right)}\right. \\
& \left.\quad+\int_{0}^{t}\left(\left\|\nabla_{x} \vartheta\right\|_{H^{d-1}\left(\mathbb{R}^{3}\right)}^{2}+\left\|\nabla_{x} E_{r}\right\|_{H^{d-1}\left(\mathbb{R}^{3}\right)}^{2}+\left\|\nabla_{x} \vec{u}\right\|_{H^{d-1}\left(\mathbb{R}^{3}\right)}^{2}+\|H\|_{H^{d-1}\left(\mathbb{R}^{3}\right)}^{2}\right)\right) .
\end{aligned}
$$

In order to conclude, we need to estimate the perturbation $H$. For this purpose, we use that $H^{d-1}\left(\mathbb{R}^{3}\right)$ is an algebra: for any $s \leq t$,

$$
\|H(s)\|_{H^{d-1}\left(\mathbb{R}^{3}\right)} \leq C N(t)\left\|\nabla_{x} V\right\|_{H^{d-1}}\left(\mathbb{R}^{3}\right) .
$$

Inserting this into (5.26), we prove (5.18).

We are now in position to conclude with the

Proof of Theorem 3.2: We first point out that local existence for system (1.4)-(1.5)-(1.6) may be proved using standard fix-point methods. We refer to [26] for the proof. The existence is proved in the following functional space:

$$
X(0, T)=\left\{V, \quad V-\bar{V} \in C\left([0, T] ; H^{d}\left(\mathbb{R}^{3}\right)\right), \begin{array}{r}
\nabla_{x} V \in L^{2}\left([0, T] ; H^{d-1}\left(\mathbb{R}^{3}\right)\right), \\
\\
\left.\nabla_{x} \vartheta, \nabla_{x} E_{r} \in L^{2}\left([0, T] ; H^{d}\left(\mathbb{R}^{3}\right)\right)\right\} .
\end{array}\right.
$$


In order to prove global existence, we argue by contradiction, and assume that $T_{c}>0$ is the maximum time existence. Then, we necessarily have

$$
\lim _{t \rightarrow T_{c}} N(t)=+\infty
$$

where $N(t)$ is defined by (5.11). We are thus reduced to prove that $N$ is bounded. For this purpose, we use the method of [24], which was also used in [28]. First note that, due to Proposition 5.4 on the one hand, and to Proposition 5.5 on the other hand, we know that there exists a non-decreasing continuous function $C: \mathbb{R}^{+} \rightarrow \mathbb{R}^{+}$such that

$$
\forall T \in\left[0, T_{c}\right], \quad N(t)^{2} \leq C(N(t))\left(N(0)^{2}+N(t)^{3}\right)
$$

Hence, setting $N(0)=\varepsilon$, we have

$$
\frac{N(t)^{2}}{\varepsilon^{2}+N(t)^{3}} \leq C(N(t)),
$$

Studying the variation of $\phi(N)=N^{2} /\left(\varepsilon^{2}+N^{3}\right)$, we see (figure 1) that $\phi^{\prime}(0)=0$, that $\phi$ is increasing on the interval $\left[0,\left(2 \varepsilon^{2}\right)^{1 / 3}\right]$ and decreasing on the interval $\left[\left(2 \varepsilon^{2}\right)^{1 / 3},+\infty\right)$. Hence,

$$
\max \phi=\phi\left(\left(2 \varepsilon^{2}\right)^{1 / 3}\right)=\frac{1}{3}\left(\frac{2}{\varepsilon}\right)^{2 / 3} .
$$

Hence, the function $C$ being independent of $\varepsilon$, we can choose $\varepsilon$ small enough to have $\phi(N) \leq C(N)$ for all $N \in\left[0, N^{*}\right]$, where $N^{*}>0$. Since $C$ is continuous, (5.28) implies that $N \leq N^{*}$. This is clearly in contradiction with (5.27).

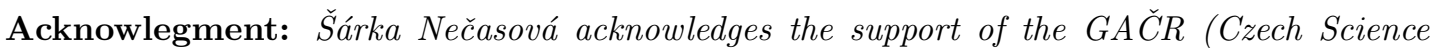
Foundation) project P201-13-00522S in the framework of RVO: 67985840.

\section{References}

[1] S. Alinhac, P. Gérard. Opérateurs pseudo-différentiels et théorème de Nash-Moser. InterEditions/Editions du CNRS, 1991.

[2] R. Balian. From microphysics to macrophysics. Methods and applications of statistical physics Vol. II. Springer Verlag, Berlin, Heidelberg, New York, 1992.

[3] C. Beauchard, E. Zuazua. Large time asymptotics for partially dissipative hyperbolic systems. Arch. Rational Mech. Anal., 199:177-227, 2011.

[4] S. Benzoni-Gavage, D. Serre. Multi-dimensional hyperbolic partial differential equations. Oxford Science Publications, 2007.

[5] C. Buet, B. Després. Asymptotic analysis of fluid models for the coupling of radiation and hydrodynamics. Journal of Qantitative Spectroscopy and Radiative Transfer, 85:385-418, 2004.

[6] S. Chandrasekhar. Radiative transfer. Dover Publications, Inc., New York, 1960.

[7] G.Q. Chen, D. Wang. The Cauchy problem for the Euler equations for compressible fluids. in "Handbook of Mathematical Fluid Dynamics, Vol. 1", S. Friedlander, D. Serre Eds. NorthHolland, Elsevier, Amsterdam, Boston, London, New York, 2002. 
[8] B. Dubroca, J.-L. Feugeas, Etude théorique et numérique d'une hiérarchie de modéles aux moments pour le transfert radiatif, C. R. Acad. Sci. Paris, 329 (1999) 915-920.

[9] B. Dubroca, M. Seaïd, J.-L. Feugeas, A consistent approach for the coupling of radiation and hydrodynamics at low Mach number, J. of Comput. Phys. 225 (2007) 1039-1065.

[10] B. Ducomet, Š. Nečasová. Global smooth solution of the Cauchy problem for a model of radiative flow. To appear in Ann. della Scuola Norm. Sup. di Pisa.

[11] B. Ducomet, E. Feireisl, Š. Nečasová. On a model of radiation hydrodynamics. Ann. I. H. Poincaré-AN 28 (2011) 797-812.

[12] E. Feireisl and A. Novotný, Singular limits in thermodynamics of viscous fluids. Birkhauser, Basel, 2009.

[13] F. Golse, G. Allaire, Transport et diffusion. Lecture Notes, Ecole polytechnique, 2010.

[14] F. Golse, B. Perthame. Generalized solutions of the radiative transfer equations in a singular case. Commun. Math. Phys., 106:211-239, 1986.

[15] B. Hanouzet, R. Natalini. Global existence of smooth solutions for partially dissipative hyperbolic systems with a convex entropy. Arch. Ration. Mech. Anal., 69(2):89-117, 2003.

[16] L. Hsiao. Quasilinear hyperbolic systems and dissipative mechanisms. World Scientific, Singapore, New Jersey, London, Hong Kong, 1997.

[17] P. Jiang, Y. Wang. Existence of solutions to an initial-boundary value problem of multidimensional radiation hydrodynamics. Journal of Differential Equations, 251:1616-1636, 2011.

[18] P. Jiang, D. Wang. Formation of singularities of solutions to the three-dimensional EulerBoltzmann equations in radiation hydrodynamics. Nonlinearity, 23:809-821, 2010.

[19] P. Jiang, Y. Wang. Global weak solutions to the Euler-Boltzmann equations in radiation hydrodynamics. Journal of Hyperbolic Differential Equations, 9:711-738, 2012.

[20] R. E. Kalman, P. L. Falb, M. A. Arbib. Topics in mathematical system theory McGraw-Hill Book Co., New York-Toronto, Ont.-London, 1969.

[21] S. Kawashima, Y. Nikkuni, S. Nishibata, Large-time behavior of solutions to hyperbolicelliptic coupled systems, Arch. Ration. Mech. Anal., 170(4), 297-329, 2003.

[22] C. Lin. Mathematical analysis of radiative transfer models. PhD Thesis, 2007.

[23] C. Lin, J. F. Coulombel, T. Goudon, Shock profiles for non-equilibrium radiative gases, Physica D, 218 (2006) 83-94.

[24] C. Lin, T. Goudon. Global existence of the equilibrium diffusion model in radiative hydrodynamics. Chin. Ann. Math., 32B:549-568, 2011.

[25] R.B. Lowrie, J.E. Morel, J.A.. Hittinger. The coupling of radiation and hydrodynamics. The Astrophysical Journal, 521:432-450, 1999.

[26] A. Majda. Compressible fluid flow and systems of conservation laws in several variables. Springer-Verlag, New-York, Berlin, Heidelberg, Tokyo, 1984.

[27] D. Mihalas, B. Weibel-Mihalas. Foundations of radiation hydrodynamics. Oxford University Press, New York, 1984. 
[28] T. Nishida, Nonlinear hyperbolic equations and related topics in fluid dynamics, Publications Mathématiques d'Orsay, No. 78-02, Département de Mathématique, Université de Paris-Sud, Orsay, 1978.

[29] G.C. Pomraning. Radiation hydrodynamics. Dover Publications, Inc., Mineola, New York, 2005.

[30] D. Serre. Systèmes de lois de conservation I, II. Diderot Editeur, Arts et Sciences, Paris, New-York, Amsterdam, 1996.

[31] D. Serre. Systems of conservation laws with dissipation. Lecture Notes SISSA, 2007.

[32] Y. Shizuta, S. Kawashima. Systems of equation of hyperbolic-parabolic type with application to the discrete Boltzmann equation. Hokkaido Math. J., 14:249-275, 1985.

[33] I. Teleaga, M. Seaïd, Simplified radiative models for low Mach number reactive flows, Appl. Math. Modeling 32 (2008) 971-991.

[34] I. Teleaga, M. Seaïd, I. Gasser, A. Klar, J. Struckmeier, Radiation models for thermal flows at low Mach number, J. of Comput. Phys. 215 (2006) 506-525.

[35] X. Zhong, S. Jiang. Local existence and finite-time blow-up in multidimensional radiation hydrodynamics. J. Math fluid mech., 9:543-564, 2007.

\section{Xavier Blanc}

Univ. Paris Diderot, Sorbonne Paris Cité,

Laboratoire Jacques-Louis Lions,

UMR 7598, UPMC, CNRS, F-75205 Paris, France

E-mail: blanc@ann.jussieu.fr

\section{Bernard Ducomet}

CEA, DAM, DIF

F-91297 Arpajon, France

E-mail: bernard.ducomet@cea.fr

Š́rka Nečasová

Institute of Mathematics of the Academy of Sciences of the Czech Republic

Žitná 25, 11567 Praha 1, Czech Republic

E-mail:matus@math.cas.cz 